Reducing the effect of seismic noise in LIGO searches by targeted veto generation

This article has been downloaded from IOPscience. Please scroll down to see the full text article.

2012 Class. Quantum Grav. 29055006

(http://iopscience.iop.org/0264-9381/29/5/055006)

View the table of contents for this issue, or go to the journal homepage for more

Download details:

IP Address: 131.215.220.186

The article was downloaded on 16/03/2012 at 16:23

Please note that terms and conditions apply. 


\title{
Reducing the effect of seismic noise in LIGO searches by targeted veto generation
}

\author{
D M Macleod ${ }^{1}$, S Fairhurst ${ }^{1}$, B Hughey ${ }^{2}$, A P Lundgren ${ }^{3,4}$, \\ L Pekowsky ${ }^{4}$, J Rollins ${ }^{5}$ and J R Smith ${ }^{4,6}$ \\ ${ }^{1}$ Cardiff University, Cardiff CF24 3AA, UK \\ ${ }^{2}$ University of Wisconsin-Milwaukee, Milwaukee, WI 53201, USA \\ 3 The Pennsylvania State University, University Park, PA 16802, USA \\ ${ }^{4}$ Syracuse University, Syracuse, NY 13244, USA \\ 5 LIGO - California Institute of Technology, Pasadena, CA 91125, USA \\ ${ }^{6}$ California State University Fullerton, Fullerton CA 92831, USA \\ E-mail: duncan.macleod@astro.cf.ac.uk
}

Received 12 August 2011, in final form 15 December 2011

Published 10 February 2012

Online at stacks.iop.org/CQG/29/055006

\begin{abstract}
One of the major obstacles to the detection and study of gravitational waves using ground-based laser interferometers is the effect of seismic noise on instrument sensitivity. Environmental disturbances cause motion of the interferometer optics, coupling as noise in the gravitational wave data output whose magnitude can be much greater than that of an astrophysical signal. We present an improved method of identifying times of high seismic noise coupling by tuning a gravitational-wave burst detection algorithm to the low-frequency signature of these events and testing for coincidence with a low-latency compact binary coalescence detection algorithm. This method has been proven highly effective in removing transients of seismic origin, with $60 \%$ of all compact binary coalescence candidate events correlated with seismic noise in just $6 \%$ of analysis time
\end{abstract}

PACS numbers: 95.55.Ym, 04.80.Nn, 07.05.Kf

(Some figures may appear in colour only in the online journal)

\section{Introduction}

The Laser Interferometer Gravitational-Wave Observatory (LIGO) [1] is designed to detect and study gravitational waves of astrophysical origin. During LIGO Science Run 6 (S6), the project operated two kilometre-scale, power-recycled, Michelson interferometers in the United States, at Hanford, WA, and Livingston, LA. Together with the French-Italian Virgo [2] detector they were involved in the search for GW signals from many signals, including the coalescence of compact binary systems [3] and unmodelled burst events [4]. 
Table 1. Description of the main seismic frequency bands and their sources.

\begin{tabular}{lll}
\hline Frequency $(\mathrm{Hz})$ & Distance $(\mathrm{km})$ & Source \\
\hline $0.01-1$ & $10^{3}$ & $\begin{array}{l}\text { Distant earthquakes } \\
\text { Microseism }\end{array}$ \\
$1-3$ & $10^{1}$ & $\begin{array}{l}\text { Far anthropogenic noise } \\
\text { Close earthquakes, wind }\end{array}$ \\
$3-10$ & $10^{0}$ & $\begin{array}{l}\text { Anthropogenic noise, wind } \\
\text { Close anthropogenic noise }\end{array}$ \\
$10-100$ & $10^{-1}$ & Clone
\end{tabular}

The output of each LIGO detector is a single data stream that in general contains some combination of a GW signal and detector noise. Transient noise events (glitches) can mask or mimic astrophysical signals, thus limiting the sensitivity of any search that can be performed over these data [5-7]. In the searches for short-duration GW signals, the noise background is dominated by glitches, requiring intense effort from analysis groups and detector scientists to understand the physical origins and eliminate them. Throughout the lifetime of LIGO up to and including S6, search sensitivity has been improved by careful use of vetoes. Vetoes allow analysts to tune and operate search pipelines using a subset of cleaner data, increasing the chance of extracting a signal from the noise [8].

The detrimental effect of seismic noise has been known to be a key limiting factor to the sensitivity of GW detectors at low frequencies (below $100 \mathrm{~Hz}$ [9]). However, it is also a common cause of glitches at higher frequencies due to nonlinear coupling of low-frequency seismic noise into the gravitational wave readout. Previous methods to generate veto segments for times of high seismic noise have proven ineffective. In this paper, we introduce a new method of constructing vetoes for the specific case of seismic noise that has proven highly effective when used in the latest searches for transient GW signals. We find a large statistical correlation between triggers from the low-latency compact binary coalescence (CBC) search and seismic noise, vetoing $60 \%$ of all triggers in $6 \%$ of time for $\mathrm{H} 1$ and $6 \%$ of triggers in $0.6 \%$ of time for L1.

The paper is set out as follows. In section 2, we describe the seismic environment at each of the LIGO sites, and the effect it has on detector sensitivity. In section 3, we outline existing veto methods used in S5 and S6. In section 4, we describe our new method for identifying and vetoing noise in seismometer data. In section 5 , we present the results in terms of veto efficiency and deadtime. Finally, in section 6, we present a brief discussion of implications and further applications of the method.

\section{Seismic noise in LIGO}

\subsection{LIGO seismic environment}

The two LIGO sites were chosen to be far from urbanized areas, thus reducing the incident seismic noise, whilst their separation provides a long baseline helpful in sky localization of astrophysical signals [10]. The various types of seismic noise to which they are subject, as characterized by their source, can be separated into the four frequency bands given in table 1 , and the variability of noise during evenings and weekends relative to standard working hours is shown in figure 1 . The strain sensitivity of the two LIGO detectors is shown in figure 2, with seismic noise the limiting factor below $50 \mathrm{~Hz}$.

LIGO Hanford Observatory (LHO) is located $15 \mathrm{~km}$ from the United States Department of Energy (USDOE) Hanford site, in which several working areas include use of heavy earthmoving machinery, and the Tri-Cities area begins roughly $20 \mathrm{~km}$ away, both contributing 

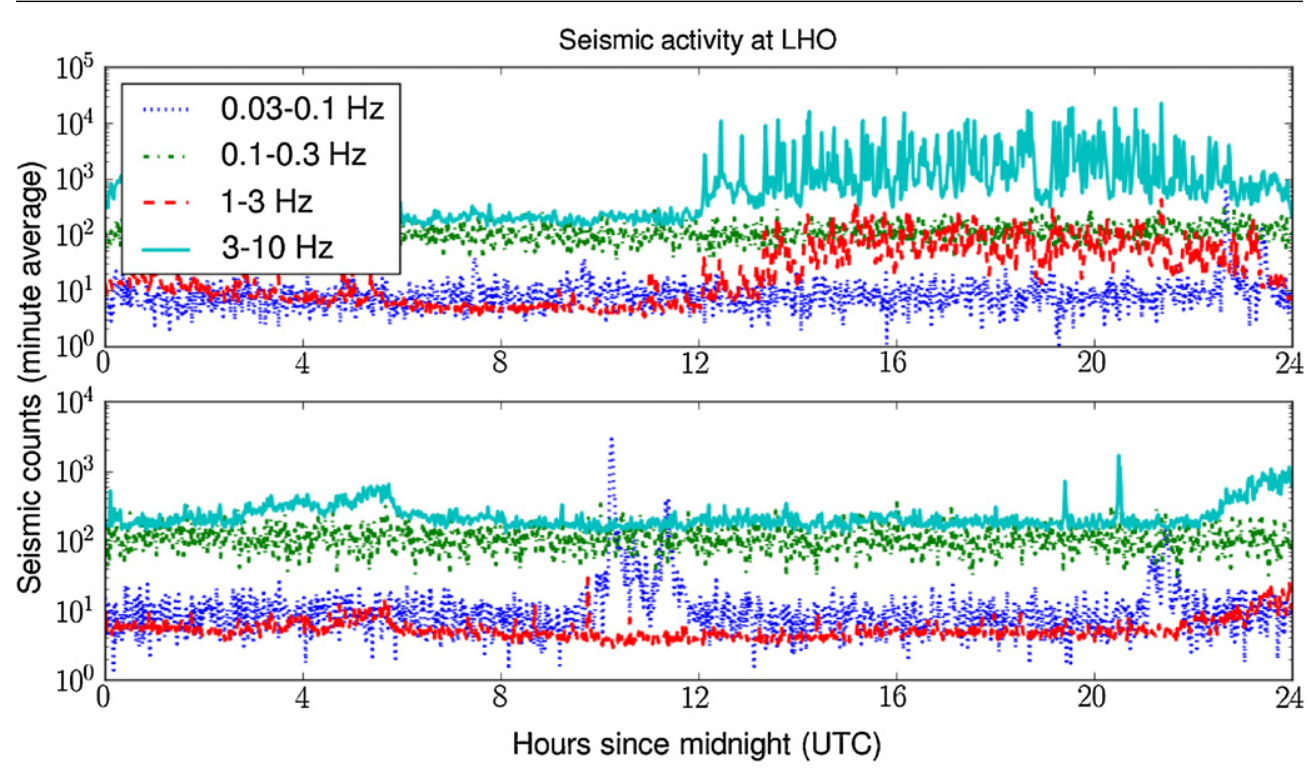

Figure 1. Examples of the difference between seismic-induced acceleration incident at the LHO site during a full $24 \mathrm{~h}$ span on a weekday (top) and a weekend (bottom), as measured by a seismometer. The higher frequency bands show elevated noise on a weekday between 12:00-24:00 UTC (05:00-17:00 local time) from working-day traffic.

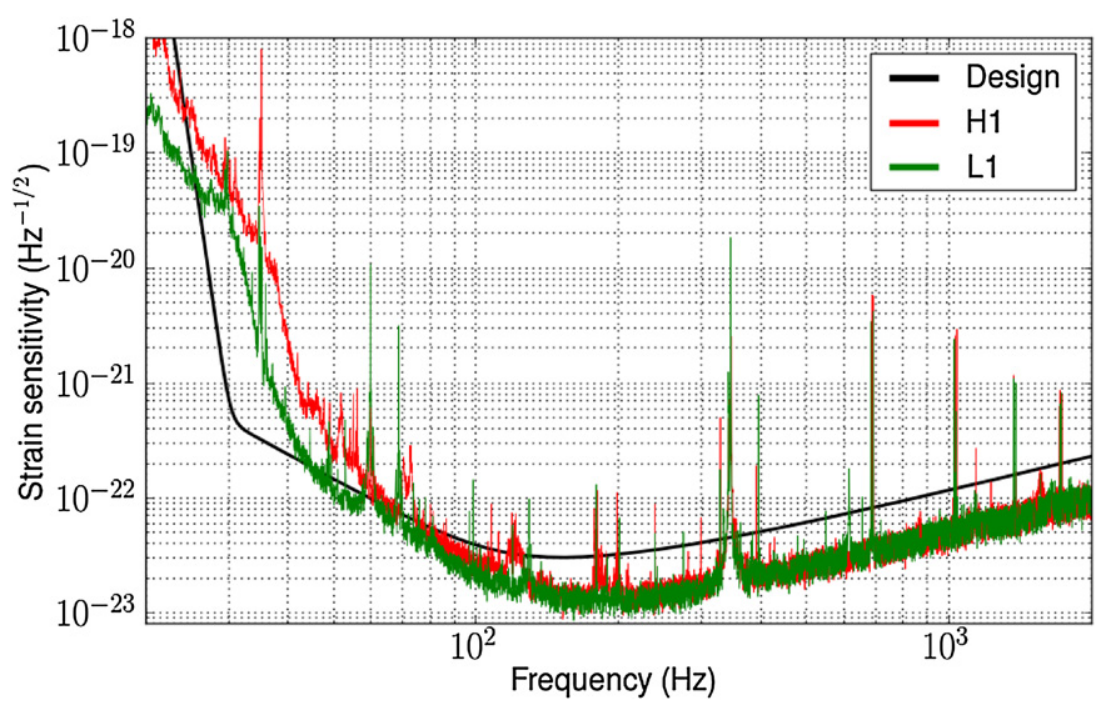

Figure 2. Strain sensitivity of the LIGO interferometers during LIGO S6. The seismic background (from direct coupling only) is the limiting noise source below $\sim 50 \mathrm{~Hz}$.

heavily to the amount of anthropogenic seismic noise incident on the detector. The site is also subject to high winds up to $40 \mathrm{~m} \mathrm{~s}^{-1}$, causing motion of the buildings and the concrete slabs supporting the instruments. These relatively local sources generate noise in the higher bands in table 1 , above $\sim 1 \mathrm{~Hz}$.

LIGO Livingston Observatory (LLO) is located $7 \mathrm{~km}$ from the town of Livingston, and only $3 \mathrm{~km}$ from a railway line used daily by cargo trains [9], plus, the land surrounding LLO 
is used for timber harvesting. The site is only $130 \mathrm{~km}$ from the Gulf of Mexico and is subject to violent rain and windstorms.

Both sites are also subject to noise from earthquakes occurring almost anywhere on the Earth, and to microseismic noise from oceanic activity due to their relatively short separation from the nearest coastline. These distant events are sources of noise below $\sim 1 \mathrm{~Hz}$.

Due to the softer composition of the surrounding geological landscape, seismic noise was worse at LLO during early science runs, so the decision was taken to install an active seismic isolation system on L1 before S4. The hydraulic external pre-isolator (HEPI) feedforward system damps low-frequency noise by using signals from the onsite seismometers to control movement of the vacuum chambers for the end test masses. This particular system was not installed at LHO before S6-although other isolation systems were used — but will be installed as part of the Advanced LIGO (aLIGO) project [11].

The LIGO instruments are designed to be sensitive in the range 40-7000 $\mathrm{Hz}$ [1], so one may be forgiven for assuming that seismic noise below $30 \mathrm{~Hz}$ should not affect sensitivity in the detection band. However, upconversion has been a problem during Initial and Enhanced LIGO, caused by a number of factors related to ground motion, e.g., scattered light [12]. This effect contaminates the sensitive band of the LIGO detectors, meaning seismic noise is an even greater problem than it would be otherwise.

\section{Existing veto methods}

The GW data stream is not the only information drawn from the LIGO detectors. Thousands of auxiliary data channels are recorded, containing control and error signals from instrumental systems, and measurements from the physical environment monitors (PEMs). These data are analysed in order to study and improve detector performance, but also to identify and remove glitches that can mimic gravitational waves. Veto segments can be constructed around excess noise if it is known to couple into the detection channel.

In S5 and S6, vetoes were constructed by two methods [5, 7, 8, 13, 14].

The first method relied on the known physical couplings between an auxiliary subsystem and the GW data, whereby when a correlation is understood, the time stream of a particular auxiliary channel is analysed, and times for which a certain threshold was exceeded are recorded. Simple, but highly effective examples include overflows in analog-to-digital converters and light dips (drops in the power stored in a detector arm cavity).

The second method replaces the known couplings with statistics, applying the KleineWelle (KW) wavelet-based algorithm [15] to data in auxiliary channels with negligible sensitivity to gravitational waves, producing lists of triggers. These events are then tested for time coincidence with triggers in the GW data, indicating whether that candidate GW event was likely to be of astrophysical origin. Vetoes are constructed around a subset of triggers in the auxiliary data chosen to maximize efficiency (the fractional number of GW triggers removed by a veto), whilst minimizing deadtime (the fractional amount of analysis time that has been vetoed).

In both methods, highly effective vetoes are those with a high ratio of efficiency to deadtime. Different implementations [13,14] were used in the searches for unmodelled bursts [16] and CBCs [17] during S5, producing comparable results [5].

\section{Targeted veto methods}

The methods described in the previous section are subject to shortcomings when applied to seismometer data. Simple thresholds have to be placed high enough to catch only the worst 
noise spikes, and so have low efficiency over weekends and evenings (around lower seismic noise as shown in figure 1). Similarly, the KW algorithm is tuned for high-frequency GWBs, with limited sensitivity to the low-frequency signature of seismic noise, resulting in low trigger numbers and poor statistical significance of coincidences.

In order to produce effective vetoes, we have devised a novel method to explicitly identify low-frequency seismic events and construct veto segments to remove this noise from GW searches. This method uses the $\Omega$-pipeline tuned specifically for low-frequency performance to generate trigger lists highlighting seismic events and the low-latency inspiral pipeline Daily iHope to generate triggers from CBC template matched filtering. The two are combined by the HierarchichalVeto algorithm into lists of time segments during which seismic noise has polluted the GW analysis.

\subsection{The $\Omega$-pipeline}

The $\Omega$-pipeline is a burst-detection algorithm developed within LIGO as a combination of the $Q$-pipeline [18] and X-pipeline [19] and used, during S6, for low-latency detection of GW events to trigger electromagnetic followup [20]. The single-detector triggers were also used for DQ investigations.

The algorithm is based on the Q transform [21] and projects detector data $s(t)$ onto a bank of windowed complex exponentials of the following form:

$$
S(\tau, f, Q)=\int_{-\infty}^{\infty} s(t) w(t-\tau, f, Q) \exp (-\mathrm{i} 2 \pi f t) \mathrm{d} t
$$

where $w$ is a time-domain window centred on $\tau, f$ is the central frequency and $Q$ is the quality factor. An example of the output of the $\Omega$-pipeline applied to low-latency gravitational wave readout data is shown in figure 3. A high density of low signal-to-noise ratio (SNR) (black) triggers is expected from Gaussian noise, but the higher SNR events (white) indicate increased noise at low frequencies, known to be correlated with the high seismic activity shown in figure 1.

As a result of using both $\mathrm{KW}$ and $\Omega$-pipeline for DQ studies, direct comparisons were drawn on the performance of each, especially in frequency reconstruction at low frequency. It was found that, in its standard implementation, the $\Omega$-pipeline gave much greater lowfrequency sensitivity and better frequency resolution.

Table 2. The parameter sets applied to the $\Omega$-pipeline search algorithm before and after tuning for low-frequency performance.

\begin{tabular}{llll}
\hline & & \multicolumn{2}{c}{ Tuned value } \\
\cline { 3 - 4 } Parameter & Untuned value & $<2 \mathrm{~Hz}$ & $>2 \mathrm{~Hz}$ \\
\hline Sample frequency & $4096 \mathrm{~Hz}$ & $4 \mathrm{~Hz}$ & $64 \mathrm{~Hz}$ \\
Frequency range & $48-2048 \mathrm{~Hz}$ & $0.01-2 \mathrm{~Hz}$ & $2-32 \mathrm{~Hz}$ \\
Block duration & $64 \mathrm{~s}$ & $65536 \mathrm{~s}$ & $4096 \mathrm{~s}$ \\
\hline
\end{tabular}

The parameter sets, as given in table 2 and summarized below, were applied to the four main seismometers at LHO-EX, EY, LVEA and VAULT ${ }^{7}$; and the three at LLO-EX, EY and LVEA (LLO has no VAULT seismometer). As can be seen in figure $4(b)$, the new parameter sets allows a huge increase in the number of triggers produced by the $\Omega$-pipeline. The density

7 The EX and EY seismometers sit outside of the vacuum chambers containing the end test masses for the $X$ - and $Y$-arms, respectively, the Large Vacuum Equipment Area (LVEA) seismometer sits beside the chamber housing the GW readout photodetector and the VAULT seismometer is in an underground chamber at a small distance from the LVEA. 


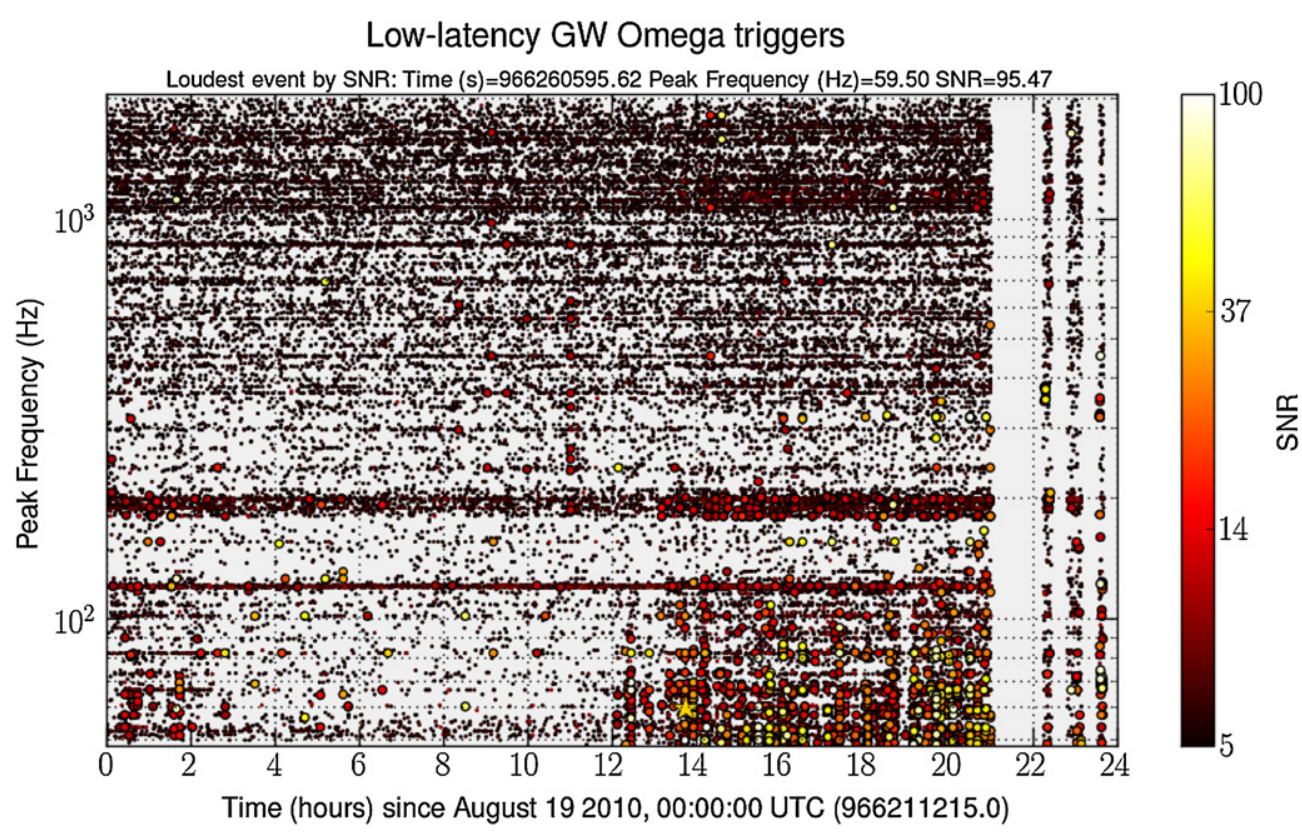

Figure 3. A $24 \mathrm{~h}$ time-frequency map from the $\Omega$-pipeline of data from the $\mathrm{GW}$ readout of the $\mathrm{H} 1$ detector for the same period as figure 1 (top). 12:00 UTC (05:00 local time) onwards subject to increased noise at the lowest frequencies due to excess seismic noise from the working day. The stripes with no events at the end of the period indicate that the detector was not operational.
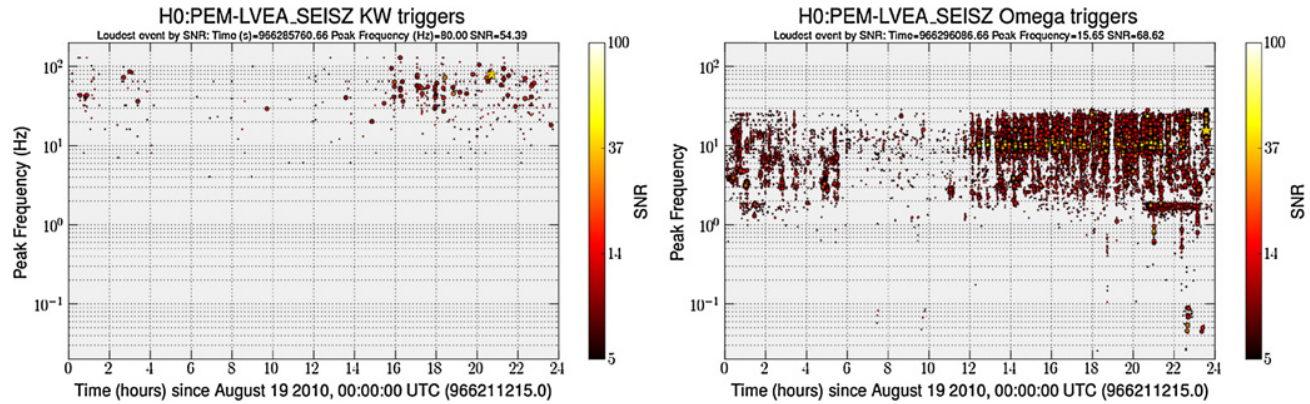

Figure 4. Examples of the untuned $\mathrm{KW}$ and tuned $\Omega$-pipeline algorithms applied to seismometer data: (a) the untuned $\mathrm{KW}$ and its relative lack of sensitivity and $(b)$ the tuned $\Omega$-pipeline with orders of magnitude with more events. Comparing to figure 3 , we can see loud triggers around 10 $\mathrm{Hz}$ after 12:00 UTC, but also triggers with the SNR above 10 below $0.1 \mathrm{~Hz}$.

of triggers has be greatly increased, especially around noisier times, with events recorded with frequencies as low as $0.03 \mathrm{~Hz}$.

\subsection{Parameterization of the $\Omega$-pipeline for seismic noise}

The low-latency $\Omega$-pipeline analysis used a parameter set tuned for performance in the detection band, with a frequency range of $48-2048 \mathrm{~Hz}$, and analyses performed using $64 \mathrm{~s}$ long data segments to estimate the background noise spectrum. As can be seen in 
figure 3, the frequency range is such that the seismic band is almost completely ignored. However, a significant SNR is recorded up to around $200 \mathrm{~Hz}$ that can be attributed, by time coincidence, to seismic noise upconversion.

In order to improve performance when applied to seismometer data, the parameter space was split into two sets: the anthropogenic band, above $2 \mathrm{~Hz}$, and very low frequency seismic activity, below $2 \mathrm{~Hz}$. The following paragraphs detail the changes made to tune the $\Omega$-pipeline algorithm for each frequency band, describing three key parameters. The sampling rate defines the highest frequency (half the sample rate) of the data to be filtered, and the frequency range gives the complete span of frequencies searched. The block duration defines the length of period used to estimate the power spectral density (PSD) of the detector, set as a power of 2 (for ease of the fast Fourier transform (FFT) computation. In order that a small number of loud events do not affect the measurement of the background, we use a duration significantly longer than the longest resolvable events and use a median-mean average method to accurately measure the background noise.

4.2.1. The anthropogenic band, $>2 \mathrm{~Hz}$. As described in section 2, the seismic band extends upwards in frequency to a few tens of Hz. Short, high-frequency events may corrupt the calculation of the background around them for a longer time, shadowing lower frequency, lower amplitude events. Lowering the sampling frequency to $64 \mathrm{~Hz}^{8}$ filtered out any high frequency seismic noise, allowing longer time-scale events to be triggered by the search. The power spectrum was drawn from blocks of $4096 \mathrm{~s}$, meaning a number of discrete seismic events above $2 \mathrm{~Hz}$ could be individually resolved above the background.

4.2.2. The earthquake band, $<2 \mathrm{~Hz}$. This band was chosen specifically to target longdistance earthquakes, that, as described in table 1 , add noise down to $0.01 \mathrm{~Hz}$ for up to several hours. Here, the sampling frequency could be reduced to $4 \mathrm{~Hz}$, eliminating higher frequency disturbances, with a minimum frequency of $0.01 \mathrm{~Hz}$, while blocks of $65536 \mathrm{~s}$ were used to allow accurate PSD estimation in the presence of hour-long earthquake events.

\subsection{Low-latency inspiral triggers-Daily iHope}

The joint LIGO-Virgo CBC group uses the iHope pipeline to search for gravitational waves produced by binary coalescences. It is described more fully in [17, 22]. Seismic noise has been known to contribute significantly to the noise background estimates in these searches, and so creating good vetoes specifically for $\mathrm{CBC}$ searches was a major goal of this work. Here, we summarize the key points and discuss the changes made for daily running of iHope in order to provide triggers to analyse alongside the $\Omega$-pipeline triggers from seismic data.

Daily iHope is a templated, matched-filter search using restricted, stationary phase, frequency-domain waveforms of the form

$$
\tilde{h}(f ; M, \eta)=\frac{2 G M_{\odot}}{c^{2} r}\left(\frac{5 M \eta}{96 M_{\odot}}\right)^{\frac{1}{2}}\left(\frac{M}{\pi^{2} M_{\odot}}\right)^{\frac{1}{3}} f^{-\frac{7}{6}}\left(\frac{G M_{\odot}}{c^{3}}\right)^{-\frac{1}{6}} \mathrm{e}^{\mathrm{i} \Psi(f ; M, \eta)},
$$

where $M=m_{1}+m_{2}$ is the total mass of the binary and $\eta=m_{1} m_{2} / M^{2}$ is the symmetric mass ratio. A static bank of such templates spanning the total mass range from $2 M_{\odot}-25 M_{\odot}$ was used for each interferometer, based on the layout at a quiet time in each instrument, with a minimal match of 0.95 for the region above a chirp mass $\left(M \eta^{3 / 5}\right)$ of $3.46 M_{\odot}$ and

8 The $\Omega$-pipeline search for gravitational waves downsamples the readout data to $4096 \mathrm{~Hz}$, while the seismometers are only sampled at $256 \mathrm{~Hz}$ 


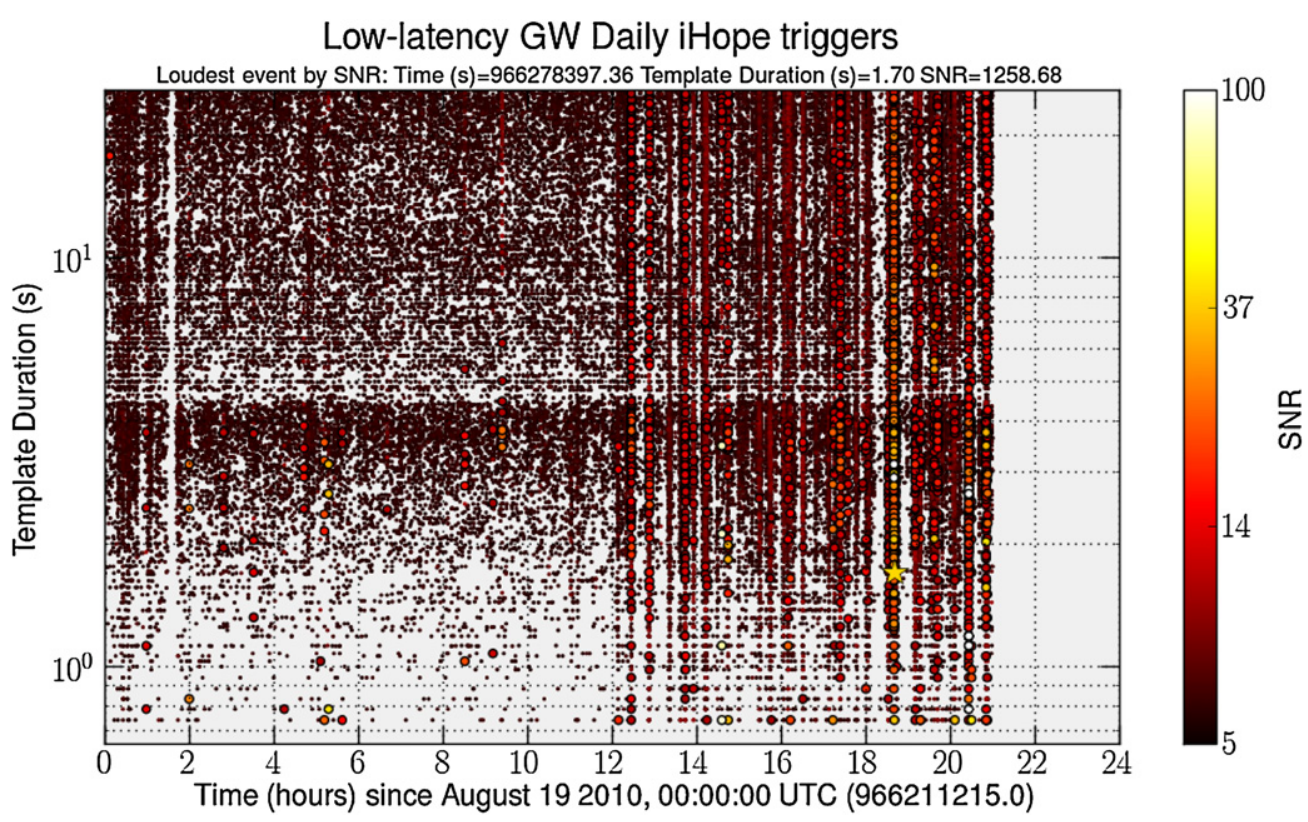

Figure 5. A $24 \mathrm{~h}$ template duration versus time map of triggers from the Daily iHope pipeline. CBC templates are better characterized by a duration rather than a frequency, as they sweep through a range of frequencies. Comparing to figures 1 and 3 , we see the same excess of triggers after 12:00 UTC, but here the excess noise at low frequency results in triggers across the entire template bank. No data are analysed after 21:00 UTC because iHope requires at least 2048 contiguous seconds to estimate the PSD and all data after this time were in smaller segments.

0.5 below that. This distribution would not be good enough for an astrophysical search, but was shown to be adequate for identifying short-duration glitches that match the higher mass (shorter) templates better. This allowed for short-duration glitches, corresponding to higher mass inspiral templates, to be flagged with a large SNR. An example of the output of Daily iHope is shown in figure 5 .

\subsection{Veto generation-HierarchichalVeto}

The seismic triggers from the $\Omega$-pipeline and the CBC triggers from Daily iHope were used to identify times of seismic noise using the statistical algorithm HVeto [14].

The HVeto algorithm tests the statistical significance of time coincidence between triggers from one channel, nominally the GW data channel, and those from auxiliary channels. The significance statistic is defined as

$$
S_{n}(x)=-\log _{10}\left(\sum_{x}^{\infty} P_{\text {poi }}\left(\mu, x^{\prime}\right)\right),
$$

where $x^{\prime}$ is the number of coincident events, $\mu$ is the expected number of random coincidences given the trigger rates in the two channels, $x$ is the series of non-negative integers and $P_{\text {poi }}$ is the Poisson probability distribution function. The significance is calculated for all channels in a two-dimensional space of time-coincidence window $T_{\text {win }}$ and SNR threshold ${ }^{9} \rho_{c}$.

9 Low-frequency events have a long duration whose maximum coupling time is not known. Also, many short-duration glitches in an auxiliary system can take a certain time to couple into the GW output. 
Table 3. HVeto results from coincidence between new $\Omega$-pipeline triggers from seismometer data and low-latency inspiral triggers for the LIGO detectors operating in S6. The most significant (loudest) channels for each frequency band and the cumulative statistics for the entire analysis are shown.

\begin{tabular}{|c|c|c|c|c|}
\hline Frequency band (Hz) & Loudest channel & Significance & Efficiency (\%) & Deadtime $(\%)$ \\
\hline \multicolumn{5}{|c|}{ (a) Results for $\mathrm{H} 1$} \\
\hline $0-1$ & $\mathrm{EX}$ & 1455.21 & 3.26 & 0.15 \\
\hline $1-3$ & EY & 355.37 & 3.19 & 0.71 \\
\hline $3-10$ & LVEA & 12024.98 & 22.11 & 1.24 \\
\hline $10-32$ & LVEA & 41042.78 & 35.99 & 1.04 \\
\hline \multicolumn{3}{|c|}{ Cumulative, all rounds } & 62.44 & 5.94 \\
\hline \multicolumn{5}{|c|}{ (b) Results for L1 } \\
\hline$\overline{0-1}$ & \multicolumn{2}{|c|}{ No channels passing selection criteria } & 0 & 0 \\
\hline $1-3$ & LVEA & 960.13 & 1.51 & 0.06 \\
\hline $3-10$ & LVEA & 420.55 & 0.88 & 0.06 \\
\hline \multirow[t]{2}{*}{$10-32$} & $\mathrm{EX}$ & 1601.22 & 2.29 & 0.07 \\
\hline & nulative, all round & & 6.95 & 0.60 \\
\hline
\end{tabular}

The most significant point on the $\left(T_{\text {win }}, \rho_{c}\right)$ plane is chosen for each auxiliary channel, with the loudest channel by significance selected. Veto times are constructed by generating segments of width $T_{\text {win }}$ around all triggers with the SNR above $\rho_{c}$ in that auxiliary channel. These segments are then removed from the analysis - allowing the next round to be 'won' by a (generally) different auxiliary channel containing less significant coincidences - and the procedure repeated until the significance of the loudest channel does not exceed a given stopping point. In this way, vetoes are generated hierarchically, allowing for little redundancy between different channels.

Several modifications were made to this algorithm in order to test and run on the new seismic triggers. Testing was completed in order to construct a new $\left(T_{\text {win }}, \rho_{c}\right)$ plane relevant for the long-duration events from the seismic data, spanning $0.1 \leqslant T_{\text {win }}<10 \mathrm{~s}$ and SNRs 10-300. Alongside this, as described in the caption to figure 5, modifications were made to first read and understand the new Daily iHope triggers and use the relevant new parameters.

\section{Results}

The method described above was used in the construction of the LIGO seismic veto dubbed SeisVeto for S6. Here, we present the results for a test sample of data, spanning from 26 June to 6 August 2010. The results for LHO are shown in table 3(a) and those for LLO in table 3(b) with each row giving the statistics for the most significant channel in each frequency band, in addition to the cumulative results for the entire period ${ }^{10}$.

For $\mathrm{H} 1$, the rounds contribute to give a cumulative efficiency of $62.5 \%$, with a cumulative deadtime of 5.94\%. This means that almost two-thirds of all triggers produced by the lowlatency inspiral pipeline are occurring in a small amount of time, which is coincident with high seismic noise. This statistic alone outlines the problem caused by seismic noise.

It should not be surprising that the most significant channel for the two higher frequency bands should be the LVEA seismometer. This building is closer than any other to a major road, so experiences the highest magnitude of seismic noise from traffic and close anthropogenic

${ }^{10}$ The cumulative results include all rounds passing selection criteria in each band, not just the most significant channel. 
noise, especially trucks serving the USDOE Hanford site and also houses the majority of interferometer control optics and subsystems, notably the GW readout photodetector.

For L1, we can see much lower statistical significance of the correlation between the seismic noise and the readout signal. This can be attributed in part to the improvements from the HEPI feedforward system for the Livingston instrument, but also to the different nature of the seismic environment relative to LHO. However, despite a lower efficiency, the ratio of efficiency to deadtime is still above 10 , highlighting the statistical correlation between seismic noise and low-latency CBC triggers.

\section{Summary}

In this paper, we have highlighted the problem of transient seismic noise in GW detection and presented a new method to not only identify but remove times of high noise from shortduration GW searches. We have demonstrated a highly effective veto, with a large efficiencyto-deadtime ratio, that has been crucial in removing the worst of the transient detector noise whilst leaving as much searchable time as possible. This method was applied to the searches for GWB and CBC signals in the final part of S6 and was seen to have a dramatic effect on the background [23]. aLIGO is a major upgrade program that will see the sensitive distance of the LIGO detectors increase by a factor of 10, giving a factor of 1000 in sensitive volume. This should mean regular detections of GW transients from CBC events [24]. However, it is likely that there will still be non-stationarities in the data from seismic events, and other sources. The method introduced here will allow us to remove them, increasing search sensitivity, and also gives a highly tuned means of directing site scientists to coupling noise sources in a newly commissioned machine.

Seismic noise was chosen as an obvious starting point, given the prevalence of glitches of seismic origin, and a prior lack of an effective veto method. This method can be generalized to any and all subsystems of the next generation of interferometers by tuning a GW burstdetection algorithm on the appropriate data channels and has the potential to lead to a great increase in search sensitivity as a result of the above benefits.

\section{Acknowledgments}

The authors would like to thank Gabriela Gonzalez, Jessica McIver, Greg Mendell, Laura Nuttall, and all members of the Detector Characterization group, the $\Omega$-pipeline team and the HierarchichalVeto team for discussions. DMM was supported by a studentship from the Science and Technology Facilities Council. SF was supported by the Royal Society. BH was supported by NSF grant PHY-0970074 and the UWM Research Growth Initiative. LP and AL were supported by NSF grant PHY-0847611. JRS was supported by NSF grants PHY-0854812 (Syracuse) and PHY-0970147 (Fullerton).

\section{References}

[1] Abbott B P et al 2009 LIGO: the laser interferometer gravitational-wave observatory Rep. Prog. Phys. 72076901

[2] Acernese F et al 2008 Status of Virgo Class. Quantum Grav. 25114045

[3] Abadie J et al 2010 Search for gravitational waves from compact binary coalescence in LIGO and Virgo data from S5 and VSR1 Phys. Rev. D 82102001

[4] Abadie J et al 2010 All-sky search for gravitational-wave bursts in the first joint LIGO-gEO-Virgo run Phys. Rev. D 81102001

[5] Blackburn L et al 2008 The LSC Glitch group: monitoring noise transients during the fifth LIGO science run Class. Quantum Grav. 25184004 
[6] Christensen N 2010 LIGO S6 detector characterization studies Class. Quantum Grav. 27194010

[7] Abadie J et al 2012 Characterization of the LIGO detectors during their sixth science run (in preparation)

[8] Slutsky J et al 2010 Methods for reducing false alarms in searches for compact binary coalescences in LIGO data Class. Quantum Grav. 27165023

[9] Daw E J, Giaime J A, Lormand D, Lubinski M and Zweizig J 2004 Long term study of the seismic environment at LIGO Class. Quantum Grav. 21 2255-73

[10] Fairhurst S 2009 Triangulation of gravitational wave sources with a network of detectors New J. Phys. 11123006

[11] Abbott R et al 2002 Seismic isolation for advanced LIGO Class. Quantum Grav. 19 1591-7

[12] Accadia T et al 2010 Noise from scattered light in Virgo's second science run data Class. Quantum Grav. 27194011

[13] Isogai T (the LIGO Scientific Collaboration and the Virgo Collaboration) 2010 Used percentage veto for LIGO and Virgo binary inspiral searches J. Phys.: Conf. Ser. 243012005

[14] Smith J R et al 2011 A hierarchical method for vetoing noise transients in gravitational-wave detectors Class. Quantum Grav. 28235005

[15] Chatterji S, Blackburn L, Martin G and Katsavounidis E 2004 Multiresolution techniques for the detection of gravitational-wave bursts Class. Quantum Grav. 211809

[16] Abbott B P et al 2009 Search for gravitational-wave bursts in the first year of the fifth LIGO science run Phys. Rev. D 80102001

[17] Abbott B P et al 2009 Search for gravitational waves from low mass binary coalescences in the first year of LIGO's S5 data Phys. Rev. D 79122001

[18] Chattergi S K 2005 The search for gravitational wave bursts in data from the second LIGO science run PhD Thesis (Cambridge, MA: Massachusetts Institute of Technology)

[19] Sutton P J et al 2010 X-pipeline: an analysis package for autonomous gravitational-wave burst searches New J. Phys. 12053034

[20] Adabie $\mathbf{J}$ et al 2012 Implementation and testing of the first prompt search for electromagnetic counterparts to gravitational wave transients Astron. Astrophys. at press doi:10.1051/0004-6361/201118219

[21] Brown J C 1991 Calculation of a constant $q$ spectral transform J. Acoust. Soc. Am. 89 425-34

[22] Abbott B P et al 2009 Search for gravitational waves from low mass compact binary coalescence in 186 days of LIGO's fifth science run Phys. Rev. D 80047101

[23] McIver J (for the LIGO Scientific Collaboration and the Virgo Collaboration) 2011 Data quality studies of enhanced interferometric gravitational wave detectors (in preparation)

[24] Abadie J et al 2010 Predictions for the rates of compact binary coalescences observable by ground-based gravitational-wave detectors Class. Quantum Grav. 27173001 